\title{
Kinetic simulations and photometry measurements of the E-H transition in cylindrical inductively coupled plasmas.
}

\author{
S Mattei ${ }^{1,2}, \mathrm{~K}$ Nishida ${ }^{3}, \mathrm{~S} \mathrm{Mochizuki}^{3}$, A Grudiev ${ }^{1}$, J Lettry $^{1}$, \\ M Q Tran ${ }^{2}$, A Hatayama ${ }^{3}$ \\ 1 CERN, CH-1211 Geneva 23, Switzerland \\ 2 Swiss Plasma Center, EPFL, Station 13, CH-1015 Lausanne, Switzerland \\ 3 Faculty of Science and Technology, Keio University, 3-14-1 Hiyoshi, Kohoku-ku, \\ Yokohama 223-8522, Japan \\ E-mail: stefano.mattei@cern.ch
}

\begin{abstract}
.
Inductively Coupled Plasmas (ICP) are well known to exhibit two modes of operation: a low density capacitive E-mode and a high density inductive H-mode. In this study we investigate the E-H transition in a cylindrical ICP, and show the effect of an external magnetic cusp field on the transition dynamics. The plasma is simulated by an Electro-Magnetic Particle-In-Cell Monte Carlo Collision code in order to take into account spatio-temporal variations of the plasma dynamics as well as kinetic effects. Simulations are compared to photometry measurements on the Linac4 $\mathrm{H}^{-}$ion source plasma chamber. We show that the E-H transition is characterized by strong spatial variations of the plasma parameters, with an axial plasma oscillation in E-mode followed by a centring in the coil region in H-mode. The external magnetic cusp field prevents electrons close to the wall to be accelerated and reduces the inductive power deposition in the plasma. This resulted in $\mathrm{a} \approx 50 \%$ higher current to achieve E-H transition compared to the configuration without cusp field. The results indicate possible improvements to the magnetic cusp field configuration in order to achieve optimal power transfer.
\end{abstract}

\section{Introduction}

Inductively Coupled Plasmas (ICP) are routinely used in many applications ranging from integrated circuit manufacturing to medical devices $[1,2]$. Two modes of operation are typically supported by ICPs. In the low density regime, the high voltage across the coil couples to the plasma capacitively (E-mode), while in high density the discharge is sustained by the electric field induced by the Radio Frequency (RF) current in the coil $[3]$.

Previous studies $[4,5,6]$ have shown that a sharp transition occurs between these two modes (E-H) if a sufficiently high current is driven through the coil. During the transition a sudden jump of the electron density and light emission is observed, 
accompanied by a modification of the Electron Energy Distribution Function (EEDF) [7]. Phase and space resolved optical emission spectroscopy measurements in planar ICPs showed the importance of the plasma spatial distribution in understanding the E-H transition [8]. This was also observed in Langmuir probe measurements and investigated as a function of the gas pressure [9]. Fluid simulations of the E-H transition were also performed in planar ICPs highlighting the spatial variations in the the two modes [10]. Numerical simulations of cylindrical ICPs, on the other hand, have only been performed in either E- or H-mode [11, 12], while the detailed description of the E-H transition has not yet been performed. Also, albeit external magnetic fields are commonly employed in conjunction with ICPs, their effect on the E-H transition dynamics requires further investigation. This finds particular importance in the operation of pulsed discharges, as the E-H transition occurs at the beginning of each pulse during the RF power ramp-up where an optimal power transfer is required.

In this study we investigate the E-H transition in a cylindrical ICP and show the effect of an external magnetic field on the transition dynamics. We have simulated the discharge with an Electro Magnetic Particle-In-Cell Monte Carlo Collision (EM-PICMCC) code in order to self-consistently take into account spatio-temporal variations of the plasma parameters as well as kinetic effects. The results are compared to photometry measurements performed on the plasma chamber of the Linac4 $\mathrm{H}^{-}$ion source at CERN [13]. Our goal is to determine under what conditions the discharge evolves from a low density to the E-H transition and suggest possible optimizations to the magnetic field configuration to achieve optimal power transfer.

\section{Geometry and numerical model}

Our investigation is carried out on the plasma chamber of the Linac $4 \mathrm{H}^{-}$ion source [13], which geometry is shown in figure 1. The hydrogen plasma is formed in a $\mathrm{Al}_{2} \mathrm{O}_{3}$ cylindrical chamber with diameter $48 \mathrm{~mm}$ and length $136 \mathrm{~mm}$. A 5 turn coil, surrounded by 6 ferrites, is installed around the plasma chamber and it is operated at $2 \mathrm{MHz}$ with an available peak power of $100 \mathrm{~kW}$ (maximum $300 \mathrm{~A}$ coil current). The plasma is ignited for $500 \mu \mathrm{s}$ with a repetition rate up to $2 \mathrm{~Hz}$. Hydrogen is injected by a piezo valve in pulsed mode. The magnetic configuration comprises a magnetic octupole field in Halbach configuration [14] and a dipole filter field. Three optical view ports are mounted on the back side to capture the light emitted by the plasma, pointing to: the plasma chamber axis, the center of the solenoid and the plasma electrode.

\subsection{Numerical model}

The aim of our simulations is to characterize the plasma evolution during the E$\mathrm{H}$ transition. We employ the combination of our EM-PIC-MCC, coupled with two commercial software applications for the simulation of the external electromagnetic fields - Ansys HFSS for the RF fields in vacuum and Opera VectorFields for the magnetic 


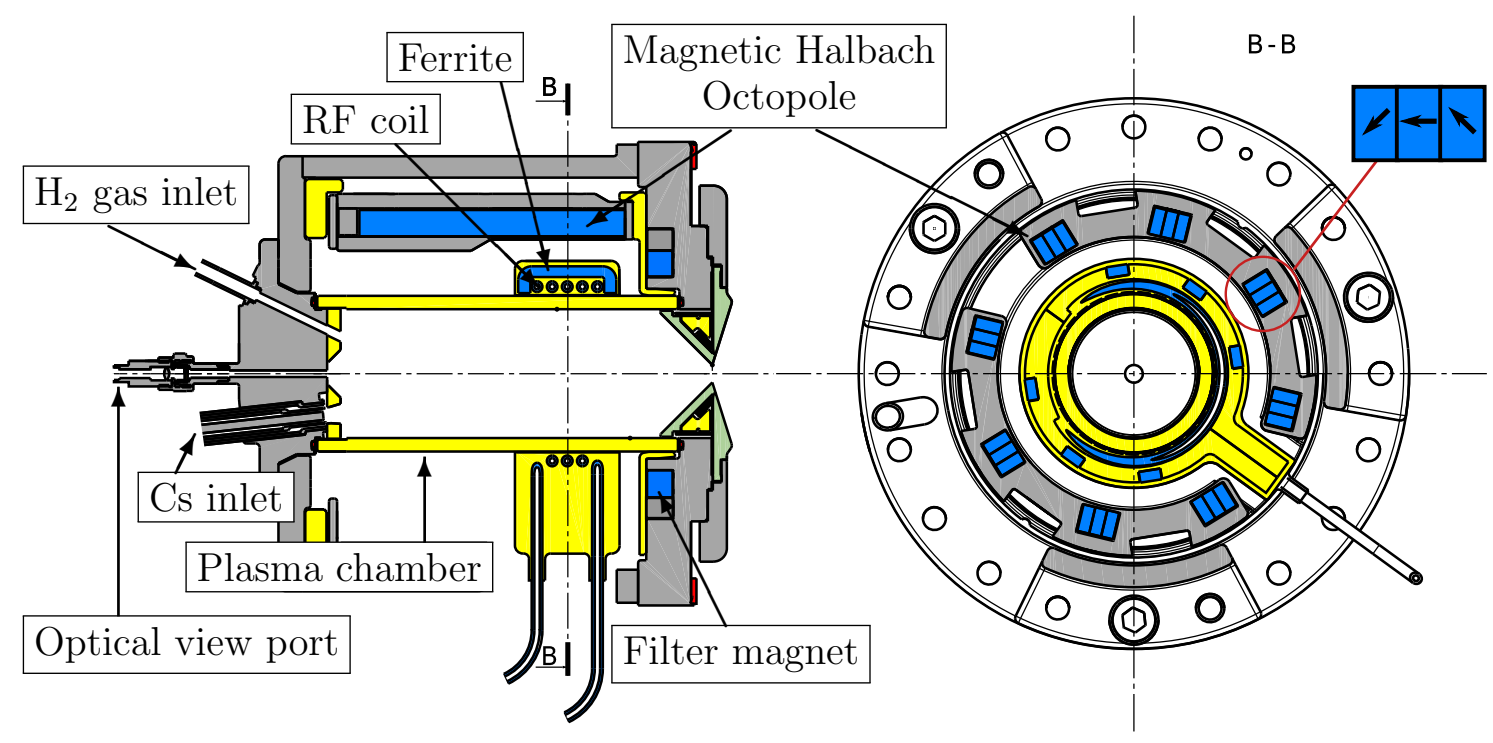

Figure 1. Longitudinal and transversal cross-section of the Linac $4 \mathrm{H}^{-}$ion source plasma generator. The plasma chamber is made of $\mathrm{Al}_{2} \mathrm{O}_{3}$. The $\mathrm{RF}$ coil is surrounded by 6 ferrites and embedded in epoxy to avoid RF breakdown. Gas is injected in pulsed mode by a piezo-valve. The optical view port has a capture angle of $3^{\circ}$. The magnetic Halbach octupole is formed by alternating magnets with clockwise and counter-clockwise magnetization. The filter field is a dipole magnet with vertical magnetization. The Cesium inlet is used to evaporate Cs onto the plasma electrode for the source operation, but it is unemployed in the present study.

octupole, described later.

The EM-PIC-MCC is a $2.5 \mathrm{D}$ code in cylindrical coordinates, meaning that the electromagnetic fields generated by the plasma $\left(\boldsymbol{E}_{\boldsymbol{p l}}, \boldsymbol{B}_{\boldsymbol{p l}}\right)$ are solved in 2D assuming axisymmetry $(\partial / \partial \theta=0)$ while the particle motion is fully $3 \mathrm{D}$. The code is the one employed in [11], supplemented with the routines to couple the external magnetic field. The simulation domain is shown in figure 2. Specifically $\boldsymbol{E}_{\boldsymbol{p l}}, \boldsymbol{B}_{\boldsymbol{p l}}$ are solved with the Finite Difference Time Domain (FDTD) method [15] with absorbing boundary conditions: Mur [16] at the axial boundary, while Bayliss and Turkel [17] at the radial boundary. The dimensions of the simulation domain are twice the plasma chamber size to avoid large reflections at the boundary. The particle motion is solved by the BorisBuneman version of the Leap-Frog method [18] and weighting to the grid is performed using a volume $\left(r^{2}-z\right)$ bilinear interpolation function with volumes treated following Verboncoeur [19]. Particles reaching the boundary of the plasma chamber are removed from the computation. The simulated plasma is composed by electrons, protons and molecular ions $\left(\mathrm{H}_{2}^{+}\right)$, initially loaded uniformly in the plasma chamber. The plasma current $\boldsymbol{j}$ required for the calculation of $\boldsymbol{E}_{\boldsymbol{p l}}, \boldsymbol{B}_{\boldsymbol{p l}}$, is the average value in the $\theta$ direction. Cell size and time step are chosen to satisfy the stability conditions of the EM-PIC 


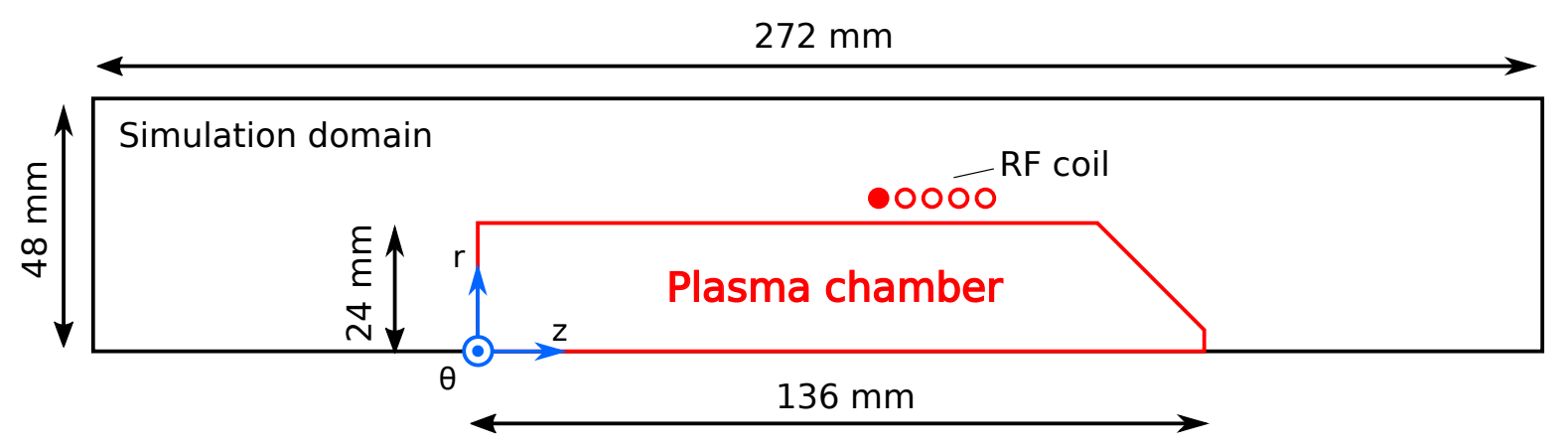

Figure 2. Simulation domain and inner dimensions of the plasma chamber. The simulation domain is twice the plasma chamber to avoid large reflections at the boundary. The RF coil position is indicated, in which the filled dot represents the high voltage connection while the opposite end is grounded.

scheme [18]. Electron-neutral and electron-ion collisions are taken into account by a Null-Collision Monte Carlo method [20] while Coulomb collisions are neglected in the present study. This choice follows since the e-neutral collision frequency is much larger than Coulomb collision one for the $\mathrm{H}_{2}$ pressures (1-10 Pa) and plasma densities $\left(<10^{16} \mathrm{~m}^{-3}\right)$ simulated [21]. The sampling of the Null collisions is performed for each cell and cross sections are taken from [22]. The neutral gas is treated as a background gas.

The RF electromagnetic fields in vacuum $\left(\boldsymbol{E}_{\boldsymbol{R} \boldsymbol{F}}, \boldsymbol{B}_{\boldsymbol{R} \boldsymbol{F}}\right)$ are simulated in the frequency domain by Ansys HFSS, starting from the ion source 3D model with its respective material properties. Details of the simulations are described in [23]. From HFSS we export a field map of $\boldsymbol{E}_{\boldsymbol{R} \boldsymbol{F}}, \boldsymbol{B}_{\boldsymbol{R} \boldsymbol{F}}$ that we average in the $\theta$ direction. Figure 3 shows the distribution of $\boldsymbol{E}_{\boldsymbol{R} \boldsymbol{F}}, \boldsymbol{B}_{\boldsymbol{R} \boldsymbol{F}}$ in the plasma chamber. The static magnetic field of the Halbach octupole $\left(\boldsymbol{B}_{\text {cusp }}\right)$ is simulated with Opera VectorFields and exported via a 3D field map for the particle dynamics model. The dipole filter field is neglected in the simulations. The computational cycle performed at each time step is illustrated in figure 4.

\section{Simulated plasma parameters}

We have performed simulations for a range of coil currents $I_{R F}$ and gas pressures $p_{H_{2}}$ as indicated in table 1 . We wish to describe the time evolution of the plasma starting from a low initial density and determine the minimum current required to achieve E$\mathrm{H}$ transition. We first describe the simulation without $\boldsymbol{B}_{\text {cusp }}$ at $p_{\mathrm{H}_{2}}=3 \mathrm{~Pa}$ and $I_{R F}=130 \mathrm{~A}$. Different conditions are later analyzed. 

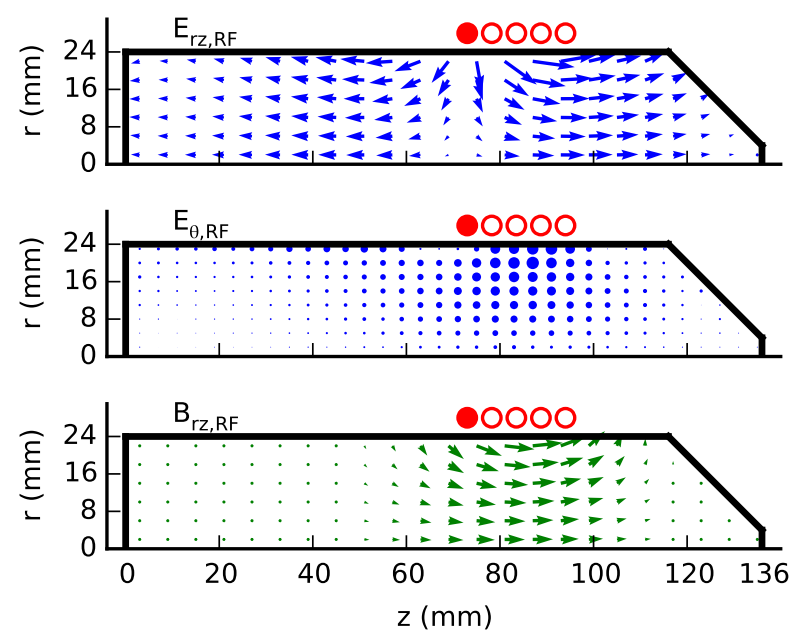

Figure 3. Distribution of the vacuum electromagnetic fields $\boldsymbol{E}_{\boldsymbol{R} \boldsymbol{F}}, \boldsymbol{B}_{\boldsymbol{R} \boldsymbol{F}}$ in the $r, z$ plane. top: $E_{r z, R F}$, middle: $E_{\theta, R F}$, bottom: $B_{r z, R F}$. The RF coil position is indicated, in which the filled dot represents the high voltage connection while the opposite end is grounded. For reference the fields for a coil current $I_{R F}=130 \mathrm{~A}$ results in maximum values of: $E_{r, R F}=45.8 \mathrm{kV} / \mathrm{m}, E_{\theta, R F}=2.1 \mathrm{kV} / \mathrm{m}, E_{z, R F}=62.5 \mathrm{kV} / \mathrm{m}$, $B_{r, R F}=9.1 \mathrm{mT}, B_{\theta, R F}=0, B_{z, R F}=16.9 \mathrm{mT}$

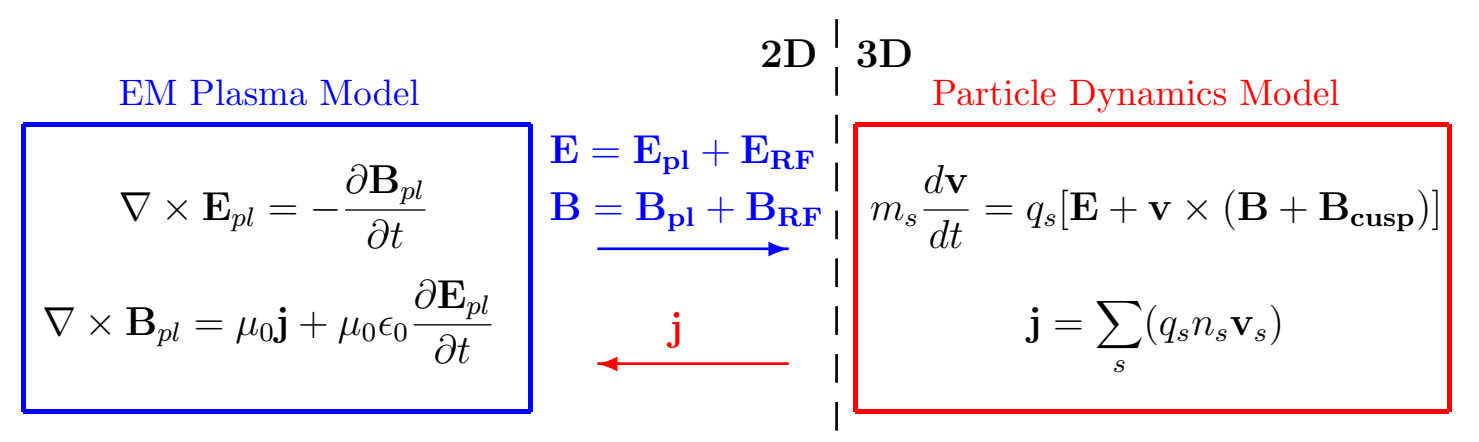

Figure 4. Computational cycle performed at each time step. The plasma current $\boldsymbol{j}$ is the sum of electrons, protons and $\mathrm{H}_{2}^{+}$contribution (index $s$ ). Weighting from the particle to the grid (and vice-versa) as required by the PIC algorithm is implied. The transition between the $3 \mathrm{D}$ to the $2 \mathrm{D}$ is performed by averaging in the $\theta$ direction

\subsection{Time evolution of the plasma}

Figure 5 shows the time variation of the average plasma density. Starting from the initial uniform density of $n_{e}=5 \times 10^{14} \mathrm{~m}^{-3}$ (chosen from previous simulations to be below the transition limit) the electron density oscillates around the initial value during the first $3 \mu \mathrm{s}$, then rapidly increases by one order of magnitude in the following $2 \mu \mathrm{s}$.

In a cylindrical ICP the azimuthal component of the electric field accounts for the inductive power deposition, while the axial and radial components are associated with the capacitive power deposition [6]. To determine the E-H transition we integrate the 
Table 1. Simulation parameters

\begin{tabular}{ll}
\hline Parameter & Value \\
\hline Cell size, $\Delta r \times \Delta z$ & $2 \times 2 \mathrm{~mm}$ \\
Time step, $\Delta t$ & $1 \mathrm{ps}$ \\
Initial particle number & 45000 \\
Real particles per simulated one & $2.5 \times 10^{6}$ \\
Initial electron density & $5.0 \times 10^{14} \mathrm{~m}^{-3}$ \\
Initial proton density & $5.0 \times 10^{13} \mathrm{~m}^{-3}$ \\
Initial $H_{2}^{+}$density & $4.5 \times 10^{14} \mathrm{~m}^{-3}$ \\
Frequency & $2 \mathrm{MHz}$ \\
Gas pressure, $p_{H_{2}}$ & $\mathbf{1}$ to $\mathbf{1 0 ~ P a}$ \\
Antenna current, $I_{R F}$ & up to $\mathbf{3 0 0 ~ A}$ \\
\hline
\end{tabular}

Joule heating power over the plasma chamber volume as a function of time and separate the capacitive component $P_{\text {cap }}$ from the inductive $P_{\text {ind }}$ :

$$
\begin{aligned}
P(t) & =P_{\text {cap }}(t)+P_{\text {ind }}(t)=\int_{V} \boldsymbol{j} \cdot \boldsymbol{E} d V \\
P_{\text {cap }}(t) & =\int_{V}\left(j_{r} E_{r}+j_{z} E_{z}\right) d V \\
P_{\text {ind }}(t) & =\int_{V} j_{\theta} E_{\theta} d V
\end{aligned}
$$

where $\boldsymbol{E}=\boldsymbol{E}_{\boldsymbol{p l}}+\boldsymbol{E}_{\boldsymbol{R} \boldsymbol{F}}$. As shown in figure 5, initially the capacitive component drives the plasma discharge being one order of magnitude larger than the inductive one. As the density increases, the inductive component rises sharply, and above $10^{15} \mathrm{~m}^{-3}$ becomes the dominant heating.

The spatial distributions of the plasma density and electromagnetic fields show significant differences in the two regimes. During the first $3 \mu \mathrm{s}$, in the low density regime, the RF axial electric field $E_{z, R F}(\approx 30$ times larger than the azimuthal component $\left.E_{\theta, R F}\right)$ drives the plasma in the axial direction as shown in figure 6 and 7 . While electrons are pulled in the direction opposite to $E_{z, R F}$, ions due to their larger inertia are only partially displaced in the direction of $E_{R F}$ during one RF cycle. This results in a spacecharge imbalance in the axial direction that generates a $E_{z, p l}$ opposite to $E_{z, R F}$. As the density increases $E_{z, p l}$ becomes equal in magnitude and opposite in direction to $E_{z, R F}$, cancelling the effect of the $\mathrm{RF}$ axial field in the plasma region. Since the plasma current exists only in a region where $E_{z}$ is weak, the capacitive power deposition drops. A similar argument applies to the radial component. However since this is only appreciable in the region close to the high voltage connection of the RF coil (figure 3), its contribution to the volume integral remains small. The azimuthal component on the other hand does not produce spacecharge imbalance given its symmetry and from this point on it is the only driving component to further heat the plasma, as shown in figure 8. 

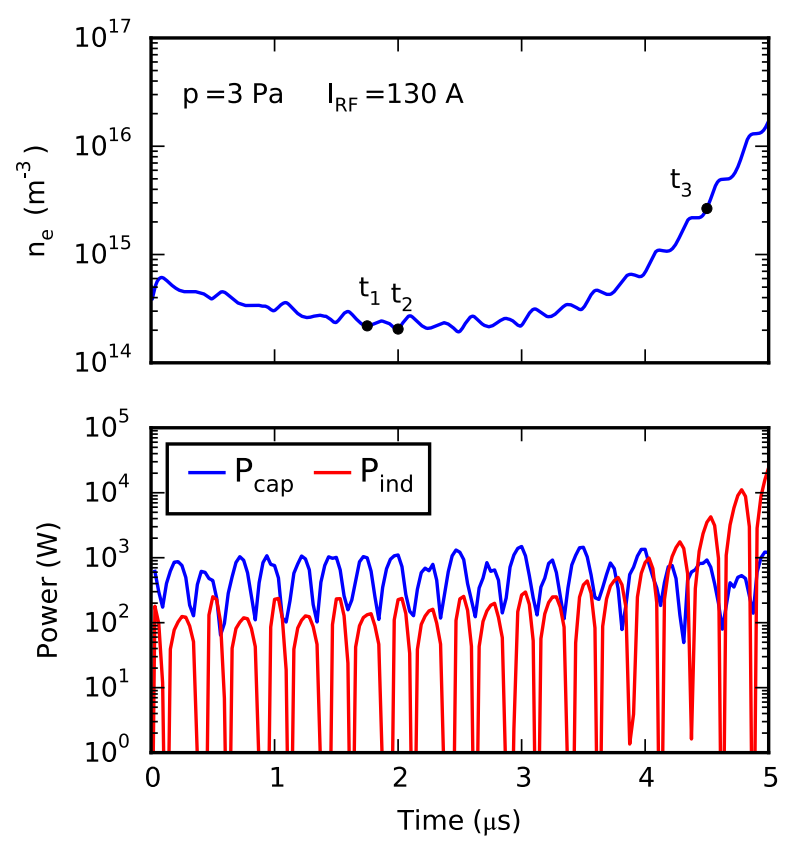

Figure 5. Average electron density (top) and Joule heating power (bottom) as a function of time. $t_{x}$ correspond to the phase of maximum $E_{R F}$. Particularly $t_{1}=1.75 \mu \mathrm{s}$ and $t_{2}=2.00 \mu \mathrm{s}$ correspond to the maximum E-field in opposite direction during the capacitively coupled regime, while $t_{3}$ is representative of the inductive coupled regime

From a numerical point of view, it is important to remark that although the initial uniform distribution represents an artificial initial condition, the plasma is strongly redistributed during the first $\mathrm{RF}$ cycle under the effect of $E_{z, R F}$ in a trend similar to figure 6 and 7 . Therefore the initial seeding distribution only influences the first RF cycle and does not perturb the remainder of the simulation. Furthermore, the plasma temperature is about $4-5 \mathrm{eV}$ in the simulations. During the initial low-density phase as well as during the E-H transition the cell size remains comparable to the Debye length $\lambda_{D e}$, avoiding numerical heating that would lead to a lack of conservation of energy. However this condition starts to be violated as the plasma density ramps to higher values in the last RF cycle. This is one of the reasons why the simulations are stopped at $5 \mu \mathrm{s}$ and the high-density steady state cannot be simulated with the chosen cell size and $\Delta t$. The computation time form $\mathrm{t}=0$ to $\mathrm{t}=5 \mu \mathrm{s}$ is 4 days on a stand-alone PC (Intel Xeon@ $2.67 \mathrm{GHz}, 16 \mathrm{~GB} \mathrm{RAM})$.

\subsection{Antenna current required for $E-H$ transition}

The E-H transition current $I_{E H}$ is defined as the value sufficient to achieve an inductive energy deposition in half RF cycle larger than the capacitive one. In other words, $I_{E H}$ 


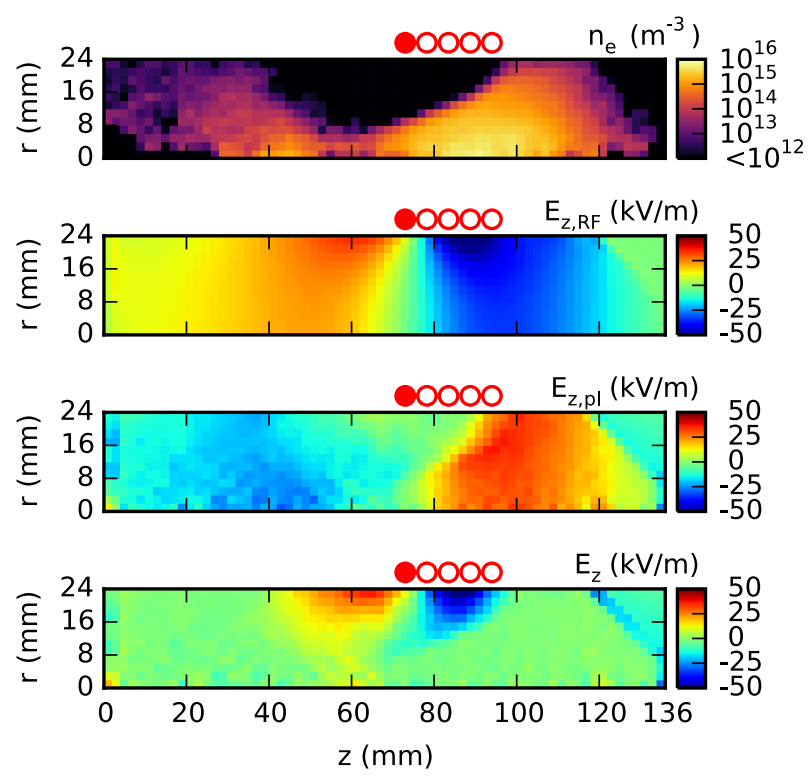

Figure 6. Distribution of the plasma parameters in the plasma chamber. From top to bottom: electron density $\left(n_{e}\right), \mathrm{RF}$ axial E-field $\left(E_{z, R F}\right)$, plasma axial E-field $\left(E_{z, p l}\right)$, total axial E-field $\left(E_{z}\right)$; evaluated at time $t_{1}$ in figure 5 . This distribution is typical for a low density plasma driven by the capacitive field at a phase of maximum E-field. The plasma responds by generating $E_{z, p l}$ opposite to $E_{z, R F}$ resulting in a total $E_{z}$ that is reduced in the plasma region

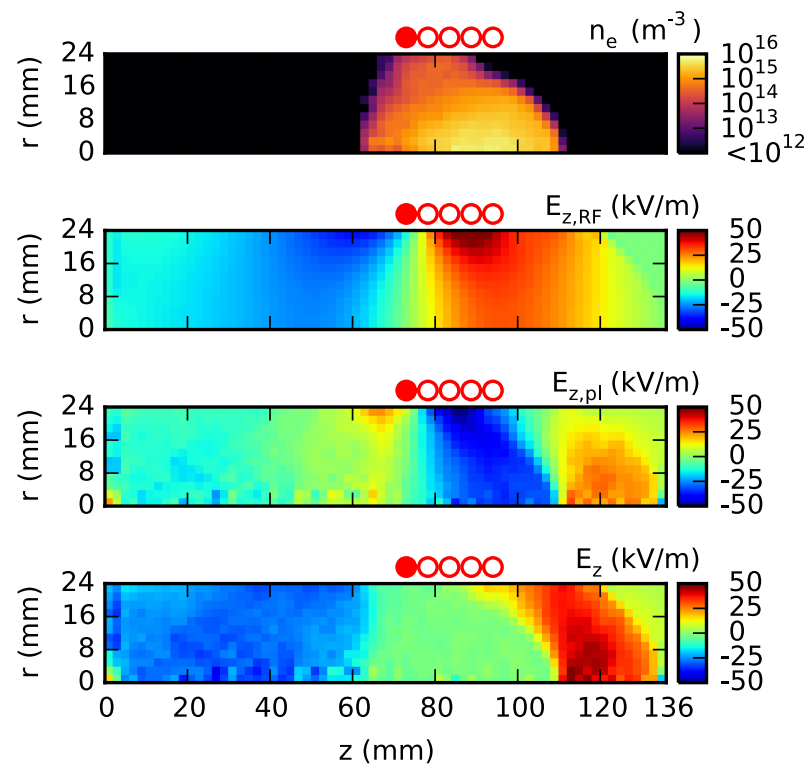

Figure 7. Distribution of the plasma parameters in the plasma chamber. From top to bottom: electron density $\left(n_{e}\right), \mathrm{RF}$ axial E-field $\left(E_{z, R F}\right)$, plasma axial E-field $\left(E_{z, p l}\right)$, total axial E-field $\left(E_{z}\right)$; evaluated at time $t_{2}$ in figure 5 . This distribution is typical for a low density plasma driven by the capacitive field at the opposite phase of maximum E-field with respect to $t_{1}$. 

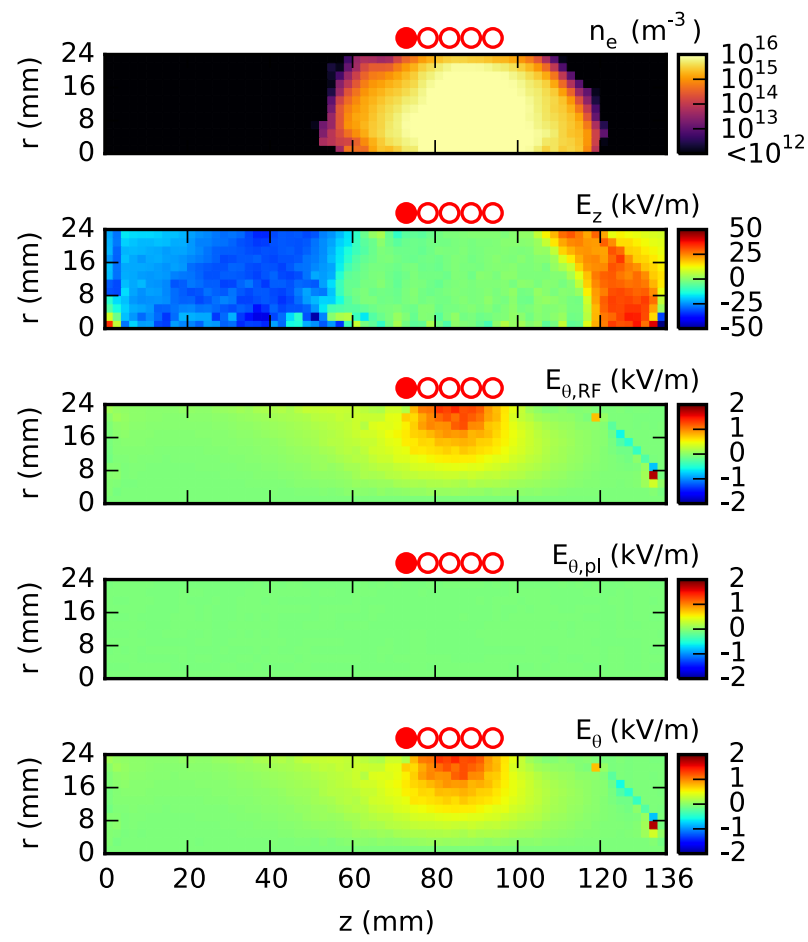

Figure 8. Distribution of the plasma parameters in the plasma chamber. From top to bottom: electron density $\left(n_{e}\right)$, total axial E-field $\left(E_{z}\right), \mathrm{RF}$ azimuthal E-field $\left(E_{\theta, R F}\right)$, plasma azimuthal E-field $\left(E_{\theta, p l}\right)$, total azimuthal E-field $\left(E_{\theta}\right)$; evaluated at time $t_{3}$ in figure 5. This distribution is representative of the density ramp-up in H-mode. While the axial field is shielded by the plasma, the azimuthal component fully penetrates into the plasma region and drives the discharge

must satisfy:

$$
\int_{t^{\prime}}^{t^{\prime}+\pi / \omega} P_{\text {ind }}(t) d t>\int_{t^{\prime}}^{t^{\prime}+\pi / \omega} P_{c a p}(t) d t
$$

where $\omega$ is the angular frequency. When this condition is met we always observe a large increase of the plasma density similar to the period around 4 us in figure 5. $I_{E H}$ depends on the neutral gas pressure with a minimum around $2 \mathrm{~Pa}$ as shown in figure 9. For pressures lower than $1.2 \mathrm{~Pa}$ the transition does not take place and the plasma extinguishes, while for higher pressures the current increases in the whole range analysed. Comparison with experimental results will be described in section 4.2.

\subsection{Simulations with cusp field}

When $B_{\text {cusp }}$ is taken into account in the simulations, a redistribution of the electrons is observed as shown in figure 10. Electrons moving towards regions of increasing azimuthal magnetic field $B_{\theta, \text { cusp }}$ are either reflected back to the plasma or trapped in the magnetic field lines leading them to the wall. 


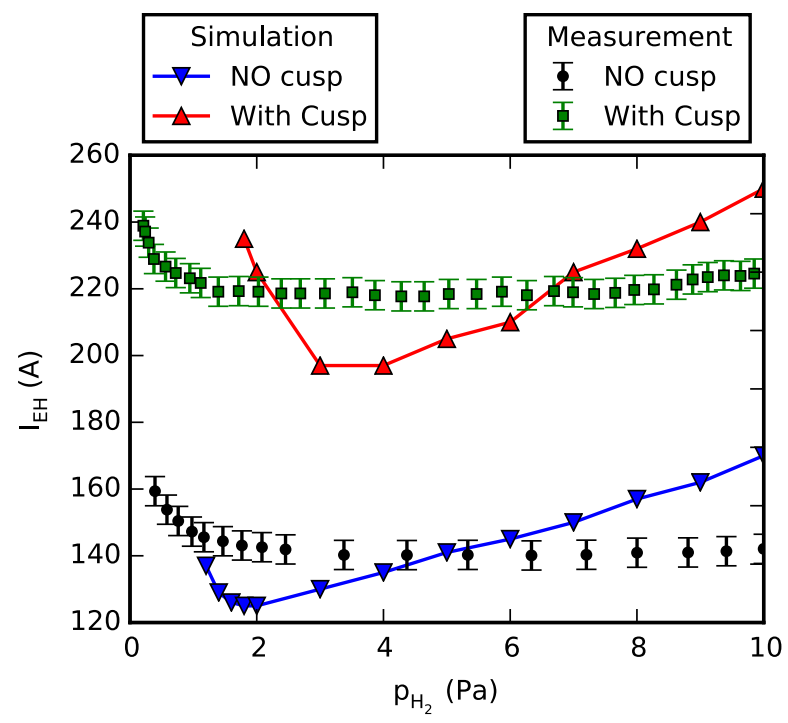

Figure 9. E-H transition current $I_{E H}$ as a function of pressure and magnetic configuration. The simulated value is the minimum current satisfying equation 4 , while the measurement corresponds to the maximum current $I_{R F}$ driven by the RF amplifier during the RF ramp-up

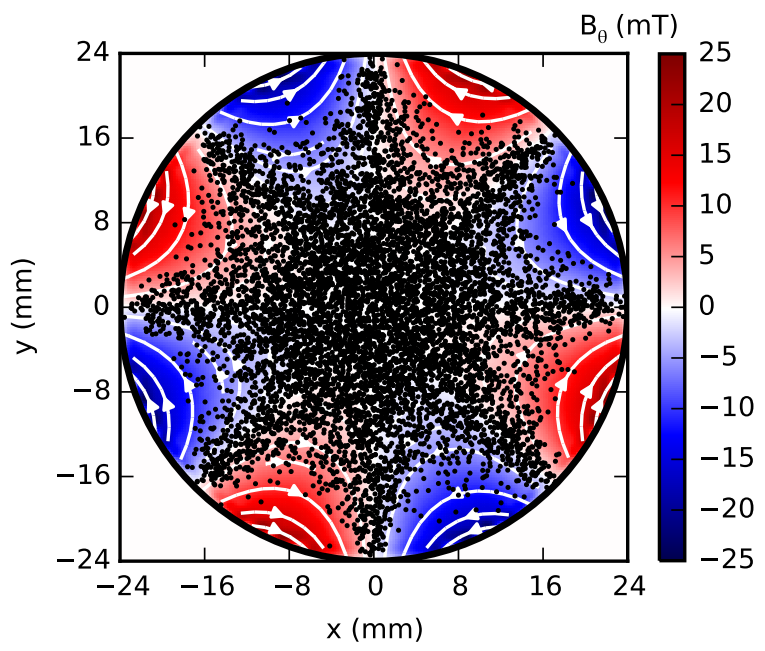

Figure 10. Simulation with magnetic cusp field. The colorbar indicates the magnitude of the $B_{\theta, \text { cusp }}$ component. White streamlines indicate the direction of $\boldsymbol{B}_{\text {cusp }}$. Electrons are represented by the black dots. The 8 loss lines typical of the magnetic octupole in Halbach configuration are clearly visible. 


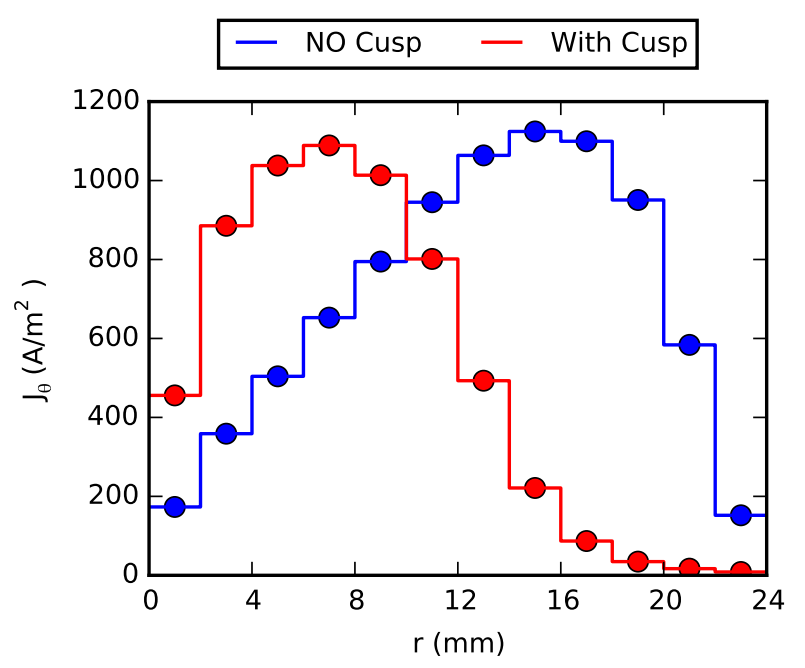

Figure 11. Average plasma current $j_{\theta}$ in the coil region during the first half cycle satisfying equation 4. Simulation conditions are $p_{\mathrm{H}_{2}}=3 \mathrm{~Pa}$ at the E-H transition current $I_{E H}=130 A$ for NO cusp and $I_{E H}=200 A$ with cusp

In this configuration $I_{E H}$ is $\approx 50 \%$ larger than the configuration without $\boldsymbol{B}_{\text {cusp }}$ (figure 9). This result is ascribed to the change in the spatial distribution of the inductive heating component resulting from the introduction of the $\boldsymbol{B}_{\boldsymbol{c u s p}}$. The inductive RF electric field $E_{\theta, R F}$ increases as a function of the radius and is therefore strongest at the radial wall (figure 8). Under the effect of $\boldsymbol{B}_{\text {cusp }}$ however, fewer electrons become available in this region to be accelerated. The plasma current remains therefore localized in the centre of the plasma chamber as shown in figure 11. In order to achieve the same inductive power deposition (equation 3) a larger $E_{\theta, R F}$ is therefore required, implying a larger $I_{R F}$.

\section{Experimental investigation}

This section describes the experimental investigation by optical emission photometry that we have performed on the Linac $4 \mathrm{H}^{-}$ion source plasma chamber. We describe the experimental setup, results and comparison to simulations.

\subsection{Experimental setup}

The experimental setup comprises 3 photomultipliers (PMT) Hamamatsu H10722-01 MOD5 to detect the plasma light emission. The light is collected by an optical fiber splitter connected to the view port on the plasma chamber axis shown in figure 1. Each fiber is directed to a PMT, whose output is read by a 12 bit oscilloscope (LeCroy HRO 64Zi). The PMT amplification gains are adjusted to cover different parts of the discharge as the light intensity typically spans 2-3 orders of magnitude. $\mathrm{PMT}_{1}$ is set to the highest gain in order to capture the first light emitted by the plasma, $\mathrm{PMT}_{2}$ is 


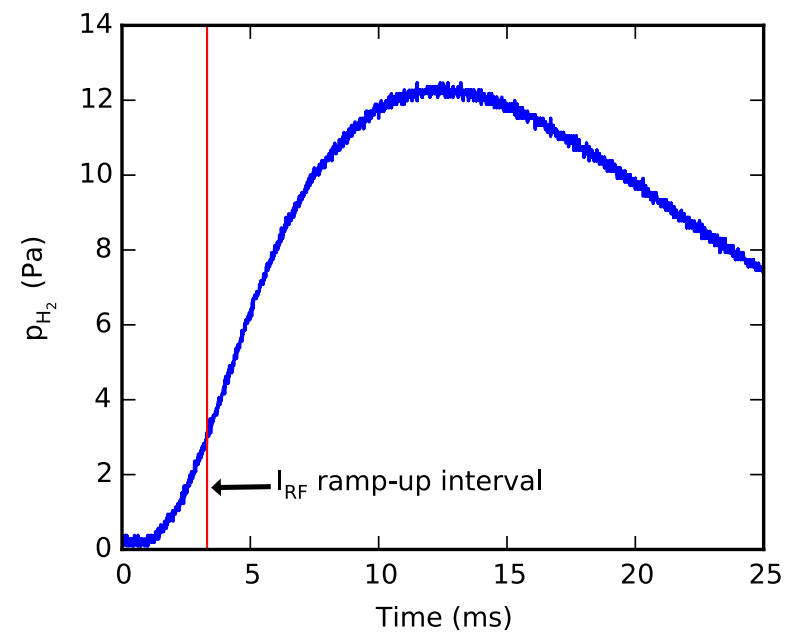

Figure 12. Pressure curve for the pulsed gas injection. The piezo-valve controlling the gas flow is opened for $500 \mu s$ at $\mathrm{t}=0$. The timing of the $I_{R F}$ ramp-up is adjusted in the range $0-10 \mathrm{~ms}$ to select the pressure during the discharge

set such that when $\mathrm{PMT}_{1}$ reaches $90 \%$ of its maximum output voltage $(3 \mathrm{~V}), \mathrm{PMT}_{2}$ is at $10 \%$. Similarly for $\mathrm{PMT}_{2}$ with respect to $\mathrm{PMT}_{3}$. This allows to reconstruct the full light signal by scaling each PMT signal by the ratio of outputs.

The RF amplifier increases $I_{R F}$ at a rate of $10 \mathrm{~A} / \mu \mathrm{s}$ up to a limit of $300 \mathrm{~A}$. All measurements are performed with the same input RF. A resonant network is employed to match the impedance to the $50 \Omega$ amplifier. $I_{R F}$ is measured by a current monitor Pearson 5046. The swift onset of the RF is synchronized to the gas injection via a piezo valve. Measurement of the pressure is taken from an off-line calibration of the pressure in the chamber as a function of the gas injection valve settings [24]. The pressure during the discharge is selected by adjusting the gas injection timing with respect to the RF ramp-up as shown in figure 12 .

\subsection{Experimental results}

Figure 13 shows the the light emission during the $I_{R F}$ ramp-up with $p_{H_{2}}=3 \mathrm{~Pa}$. As $I_{R F}$ increases a faint light is first observed for a few RF cycles $(10<\mathrm{t}<12 \mu s)$, followed by a sharp increase determining the plasma ignition. From this point the light emission enters a region characterized by asymmetric emissions with a higher peak followed by a smaller one every half RF cycle as shown in figure 14. As $I_{R F}$ further increases the light emission becomes symmetric and raises by about one order of magnitude in $2 \mu s$ (figure 15) before reaching a maximum.

For a constant requested input power $I_{R F}$ increases in $\approx 20 \mu \mathrm{s}$ from zero to a maximum value before decreasing. The maximum $I_{R F}$ driven by the $R F$ amplifier depends on the gas pressure as well as the magnetic configuration. In the presence of the magnetic cusp field the maximum current observed is a factor 1.5 higher for every 
pressure, as shown in figure 9. In both configuration the maximum $I_{R F}$ is smallest at a pressure of 2-3 $\mathrm{Pa}$, it sharply increases for lower pressures while only slightly for higher ones.

The process leading to the plasma ignition has been previously reported in the literature [25], in which the authors showed that the initial faint emission is mainly originated from molecular excitation, while the first sharp peak is due to gas dissociation leading to atomic emission. The asymmetric emission is associated with the capacitive coupling driving the plasma. As shown in [26] the line integrated light emission, reconstructed from a collision-radiative model, indicates that the higher peak results from the E-field pushing the electrons towards the view port (figure 6) while the smaller peak to the plasma centred in the plasma chamber (figure 7). The main contribution to the light intensity is the distance of the photon emission to the view port and the emission roughly scales with the local electron density. As the discharge becomes dominated by the inductive component, the plasma remains centred in the coil region (figure 8) as $E_{z, R F}$ is shielded by the plasma. As electrons are accelerated and decelerated by $E_{\theta, R F}$ the emission increases and decreases respectively giving rise to symmetric peaks. In the simulations the electron density increases by about one order of magnitude in $2 \mu s$ which corresponds to the intensity variation observed in figure 15 .

The maximum $I_{R F}$ driven by the RF amplifier correlates to $I_{E H}$ as previously reported in [6]. In the matched $\mathrm{RF}$ conditions ( $50 \Omega$ load) the $\mathrm{RF}$ amplifier increases $I_{R F}$ up to its limit of $300 \mathrm{~A}$. If a plasma is formed an extra load is added to the system that scales with the plasma density [6,27]. During the E-H transition the plasma density increases by two-three orders of magnitude, impling that the total load is no longer matched to the RF amplifier and $I_{R F}$ decreases. In other words $I_{R F}$ increases until the plasma has transited to the H-mode in which its impedance modifies the coupling efficiency and results in a drop of $I_{R F}$. The values of the maximum measured currents are reported in figure 9.

\section{Discussion}

The results highlight several important features characterizing the E-H transition dynamics. While the E-H transition is generally reported $[4,5,6]$ as an average increase of the electron density and light emission, our results indicate that its detailed description must take into account the local properties of the plasma as these vary significantly in the chamber volume as a function of time and space.

The magnetic octupole field configuration also plays a crucial role in determining the amount of power required to achieve E-H transition. While $\boldsymbol{B}_{\text {cusp }}$ can limit the wall losses, an important decrease in the ionization rate results from the spatial power deposition that is significantly reduced close to the chamber wall. This suggests that optimal power transfer can be achieved by employing a magnetic configuration that, although strong at the wall, rapidly decreases towards the centre of the plasma chamber. This can be the case of magnetic cusp fields with higher number of poles. Alternatively, 


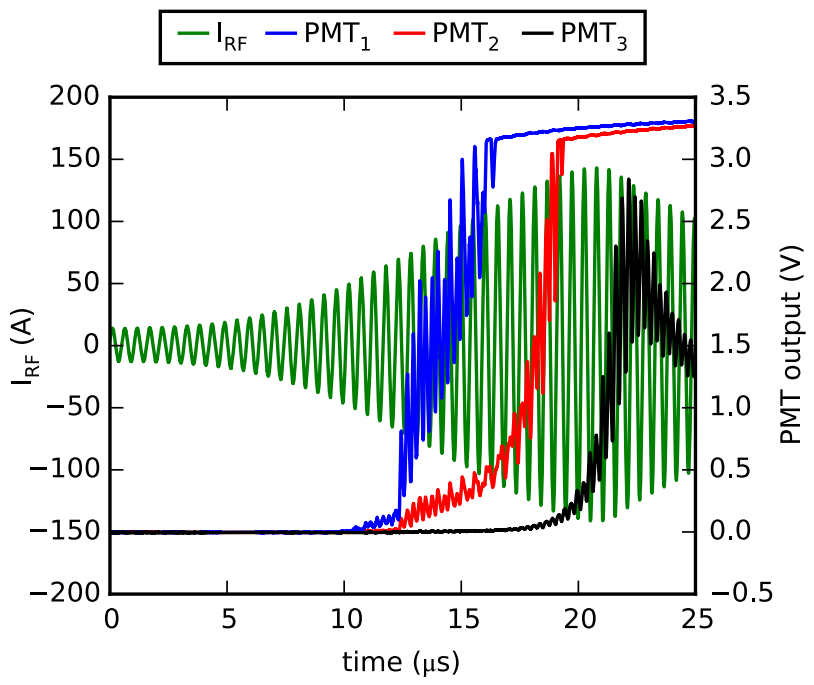

Figure 13. Sample recording of the total light emitted by the plasma during the RF current ramp-up. The PMT voltages are adjusted to cover different parts of the discharge.

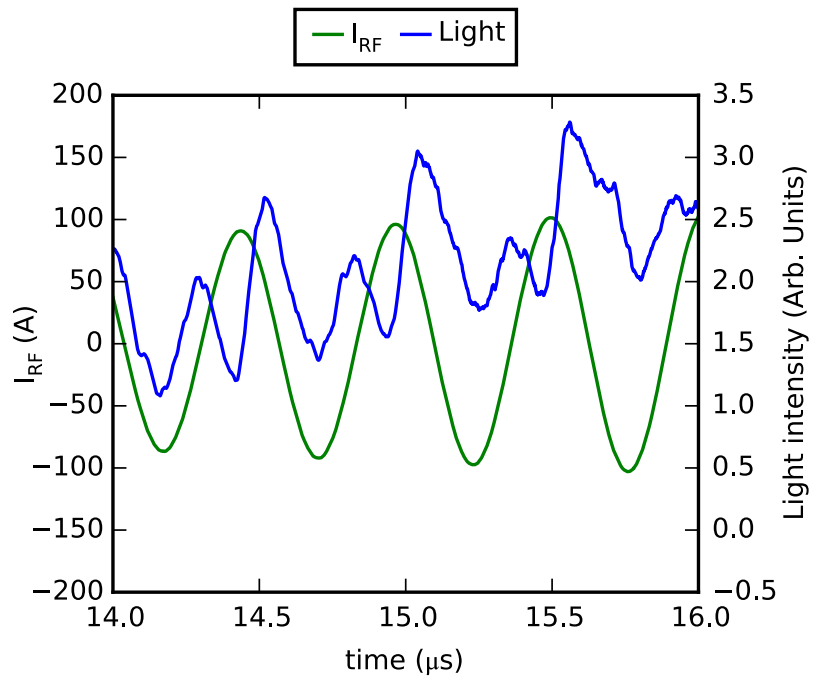

Figure 14. Measured RF current and PMT light signal $2 \mu s$ following plasma ignition. The asymmetry of the peaks intensity is characteristic of the E-mode: the higher peak is representative of the electron distribution in figure 6 , while the lower one of the distribution in figure 7

the external magnetic field can be installed to cover the plasma chamber except in the coil region. Since the azimuthal E-field is only effective in the coil region, the RF heating would not be influenced while preserving the plasma confinement in the rest of chamber.

The trend of the simulated $I_{E H}$ as a function of pressure is similar to previous experimental results $[4,6]$ and of numerical simulations in purely inductive mode [12]. As shown in [4], $\boldsymbol{E}_{\boldsymbol{R} \boldsymbol{F}}$ is most effective at transferring its energy to the electrons at a pressure for which $\nu=\omega\left(\nu\right.$ is the collision frequency, proportional to $\left.p_{H_{2}}\right)$. For pressures 


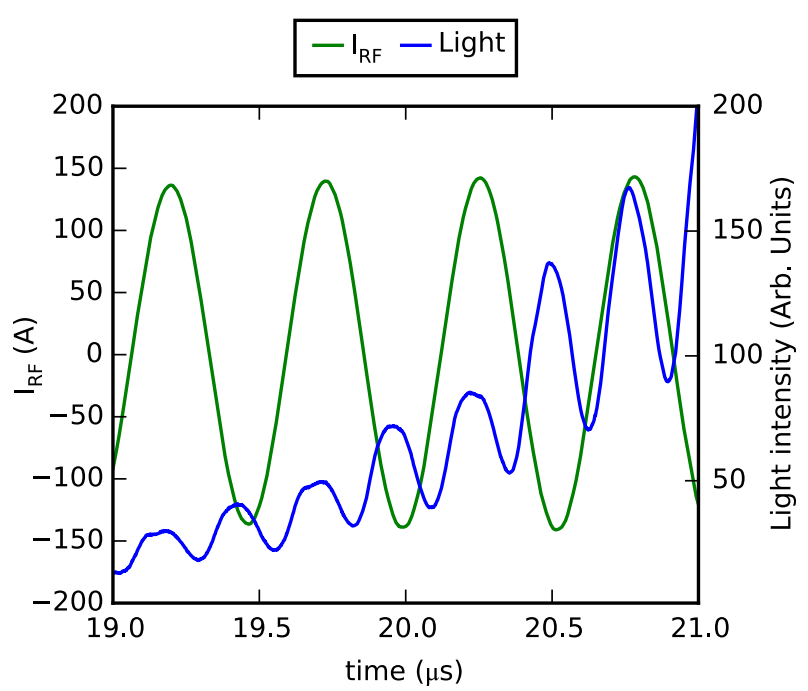

Figure 15. Light emission during the density ramp-up characteristic of a discharge transited to the H-mode. The light intensity increases by one order of magnitude in 2 $\mu s$.

below this value, the energy transfer is reduced as the electron mean free path becomes too large compared to the plasma chamber dimensions, while for higher pressures an increased $I_{E H}$ is required to compensate for the reduction in electron average kinetic energy due to a higher collision frequency [12].

The comparison of $I_{E H}$ between simulations and measurements shows two important features. First of all the range of $I_{E H}$ obtained by simulations is comparable to the one measured experimentally, both with and without $\boldsymbol{B}_{\text {cusp }}$, result validating the numerical model employed and the treatment of $\boldsymbol{B}_{\text {cusp. }}$. On the other hand the simulated $I_{E H}$ shows a different trend as a function of the hydrogen pressure compared to the measured one (figure 9). The discrepancy is similar in the case with and without $\boldsymbol{B}_{\text {cusp. }}$. We believe that the discrepancy arises due to the different conditions in which simulations and measurements are performed. A major difference between simulations and experiments is that the first are performed for a constant $I_{R F}$ starting from a given plasma distribution, while the latter are performed dynamically with a fast RF ramp-up. In the measurements we determine $I_{E H}$ based on the change of the plasma impedance occurring due the E-H transition. The plasma impedance however scales non-linearly as a function of the electron density and temperature [6], both parameters depending on the pressure. Furthermore, one of the main assumptions in the simulations is the use of a background gas with fixed dissociation degree. Since gas dissociation depends on the electron energy, a larger dissociation degree can be expected at low pressures (higher energy) and vice-versa. This would modify the collision frequency and the ionization rate due to the different cross sections of molecular and atomic processes [22]. While the method allows a qualitative investigation and to clearly identify the effect of $\boldsymbol{B}_{\text {cusp }}$, a one-to-one comparison to the simulations is beyond the reach of the study and requires 
further investigations. Possible improvements could be achieved by modelling the RF circuit together with the plasma dynamics, or the use of an experimental setup similar to [4].

\section{Conclusions and outlook}

We have simulated the E-H transition by an EM-PIC-MCC code and compared the results to photometry measurements performed on the plasma chamber of the Linac4 $\mathrm{H}^{-}$ion source. The results show that in the E-mode the plasma oscillates in the axial direction under the effect of the capacitive field. As the density increases the plasma shields the capacitive field and induction drives the heating process. We showed that the spatial plasma distribution plays a crucial role in the E-H transition dynamics.

The magnetic octupole modifies the electron distribution in the plasma chamber and reduces the power deposition in the region close to the wall. This resulted in a $\approx 50 \%$ higher current to achieve the E-H transition.

The simulations agreed well with the experiments, reproducing qualitatively the trends during the E-H transition and quantitatively the effect of the magnetic cusp field. Quantitative analysis of the pressure variation requires the implementation of a neutral transport model as well as the inclusion of the RF circuit model in the simulations. This will be the object of further studies.

\section{References}

[1] J Hopwood. Review of inductively coupled plasmas for plasma processing. Plasma Sources Science and Technology, 1(2):109, 1992.

[2] Mounir Laroussi. Low temperature plasma-based sterilization: Overview and state-of-the-art. Plasma Processes and Polymers, 2(5):391-400, 2005.

[3] Pascal Chabert and Nicholas Braithwaite. Physics of radio-frequency plasmas. Cambridge University Press, 2011.

[4] K Chandrakar. The transition from the first to the second stage of the ring discharge. Journal of Physics D: Applied Physics, 11(13):1809, 1978.

[5] AM Daltrini, SA Moshkalev, TJ Morgan, RB Piejak, and WG Graham. Plasma power measurement and hysteresis in the $\mathrm{EH}$ transition of a $\mathrm{RF}$ inductively coupled plasma system. Applied Physics Letters, 92(6):61504-61900, 2008.

[6] U Kortshagen, ND Gibson, and JE Lawler. On the E-H mode transition in RF inductive discharges. Journal of Physics D: Applied Physics, 29(5):1224, 1996.

[7] Hyo-Chang Lee and Chin-Wook Chung. Effect of electron energy distribution on the hysteresis of plasma discharge: Theory, experiment, and modeling. Scientific reports, 5, 2015.

[8] Th Wegner, C Küllig, and J Meichsner. Electron heating during eh transition in inductively coupled rf plasmas. Plasma Sources Science and Technology, 24(4):044001, 2015.

[9] Th Wegner, C Küllig, and J Meichsner. Mode transition and hysteresis in inductively coupled radio frequency argon discharge. Physics of Plasmas (1994-present), 23(2):023503, 2016.

[10] E Kawamura, M A Lieberman, A J Lichtenberg, and D B Graves. Two-dimensional simulation of inductive-capacitive transition instability in an electronegative plasma. Plasma Sources Science and Technology, 21(4):045014, 2012.

[11] S Mattei, M Ohta, M Yasumoto, A Hatayama, J Lettry, and A Grudiev. Plasma ignition 
and steady state simulations of the Linac4 $\mathrm{H}^{-}$ion source. Review of Scientific Instruments, 85(2):02B115, 2014.

[12] M Ohta, S Mattei, M Yasumoto, A Hatayama, and J Lettry. Numerical study of the inductive plasma coupling to ramp up the plasma density for the Linac4 $\mathrm{H}^{-}$ion source. Review of Scientific Instruments, 85(2):02B113, 2014.

[13] J. Lettry, D. Aguglia, J. Alessi, P. Andersson, S. Bertolo, S. Briefi, A. Butterworth, Y. Coutron, A. Dallocchio, N. David, E. Chaudet, D. Faircloth, U. Fantz, D. A. Fink, M. Garlasche, A. Grudiev, R. Guida, J. Hansen, M. Haase, A. Hatayama, A. Jones, I. Koszar, J.-B. Lallement, A. M. Lombardi, C. Machado, C. Mastrostefano, S. Mathot, S. Mattei, P. Moyret, D. Nisbet, K. Nishida, M. O'Neil, M. Paoluzzi, R. Scrivens, T. Shibata, D. Steyaert, N. Thaus, and G. Voulgarakis. Linac4 $\mathrm{H}^{-}$ion sources. Review of Scientific Instruments, 87(2), 2016.

[14] Matthias Kronberger, Elodie Chaudet, Gilles Favre, Jacques Lettry, Detlef Küchler, Pierre Moyret, Mauro Paoluzzi, Laurent PreverâLoiri, Claus Schmitzer, Richard Scrivens, and Didier Steyaert. Magnetic Cusp Configuration of the SPL Plasma Generator. AIP Conference Proceedings, 1390(1):255-264, 2011.

[15] Kane Yee. Numerical solution of initial boundary value problems involving Maxwell's equations in isotropic media. Antennas and Propagation, IEEE Transactions on, 14(3):302-307, 1966.

[16] Gerrit Mur. Absorbing boundary conditions for the finite-difference approximation of the timedomain electromagnetic-field equations. Electromagnetic Compatibility, IEEE Transactions on, EMC-23(4):377-382, 1981.

[17] Alvin Bayliss and Eli Turkel. Radiation boundary conditions for wave-like equations. Communications on Pure and applied Mathematics, 33(6):707-725, 1980.

[18] Charles K Birdsall and A Bruce Langdon. Plasma physics via computer simulation. CRC Press, 2004.

[19] J.P. Verboncoeur. Symmetric spline weighting for charge and current density in particle simulation. Journal of Computational Physics, 174(1):421 - 427, 2001.

[20] Kenichi Nanbu. Probability theory of electron-molecule, ion-molecule, molecule-molecule, and coulomb collisions for particle modeling of materials processing plasmas and gases. IEEE Transactions on plasma science, 28(3):971-990, 2000.

[21] S Mochizuki, S Mattei, T Shibata, K Nishida, A Hatayama, and J Lettry. Initial results of a full kinetic simulation of $\mathrm{RF} \mathrm{H}^{-}$source including Coulomb collision process. In FOURTH INTERNATIONAL SYMPOSIUM ON NEGATIVE IONS, BEAMS AND SOURCES (NIBS 2014), volume 1655, page 020016. AIP Publishing, 2015.

[22] Ratko K Janev, Detlev Reiter, and Ulrich Samm. Collision processes in low-temperature hydrogen plasmas. Juel-Report 4105, 2003.

[23] A Grudiev, J Lettry, S Mattei, M Paoluzzi, and R Scrivens. Numerical simulation of electromagnetic fields and impedance of CERN Linac $4 \mathrm{H}^{-}$source taking into account the effect of the plasma. Review of Scientific Instruments, 85(2):02B134, 2014.

[24] E Mahner, C Pasquino, C Schmitzer, M O'Neil, P Chiggiato, J Lettry, S Mattei, and H Neupert. Gas injection and fast pressure-rise measurements for the Linac4 $\mathrm{H}^{-}$source. In AIP Conf. Proc., volume 1515, pages 425-432, 2013.

[25] K. Chandrakar and A. Von Engel. The starting mechanism of the first stage of the ring discharge. Proceedings of the Royal Society of London A: Mathematical, Physical and Engineering Sciences, 284(1398):442-454, 1965.

[26] T Shibata, S Mattei, K Nishida, J Lettry, and A Hatayama. Numerical and experimental study of atomic transport and Balmer line intensity in Linac4 negative ion source. In FOURTH INTERNATIONAL SYMPOSIUM ON NEGATIVE IONS, BEAMS AND SOURCES (NIBS 2014), volume 1655, page 020008. AIP Publishing, 2015.

[27] K Nishida, S Mochizuki, M Ohta, M Yasumoto, J Lettry, S Mattei, and A Hatayama. Equivalent circuit of radio frequency-plasma with the transformer model. Review of Scientific Instruments, 85(2):02B117, 2014. 